\title{
Mental health after head injury
}

Suicide is associated with traumatic brain injury according to the study described by Teasdale and Engberg in this volume (pp 436-440). ${ }^{1}$ Using the opportunity for record linkage in Denmark, the authors compared suicide rates after concussion, cranial fracture, and probable brain lesion (defined as those with International Classification of Diseases codes for cerebral contusion or traumatic intracranial haemorrhage). All these groups, as expected, had higher suicide rates than the general population. Of most interest though is that those with lesion had higher suicide rates than those with concussion (hazard ratio $1.4 ; 95 \%$ confidence interval $(95 \% \mathrm{CI}) 1.2-1.8)$ and fracture (1.5; 95\% CI 1.1-2.1) after adjustment for sex, age at injury, and substance misuse.

The reliance on routine data will raise concerns about the quality of the original head injury classification. However, any random misclassification would be expected to have reduced the size of association. The strength of the study is in the comparison within the group who have received head injuries. On balance this seems to be an interesting and robust finding that deserves replication.

Suicide is an important cause of premature mortality, especially in young men. An increase in suicide rate of the size described by Teasdale and Engberg $^{1}$ will have an important impact on the health and life expectancy of those with cerebral lesion after head injury. What is the likely cause of this increase and what action should clinicians take in response to the finding?

Two possible risk factors for suicidal behaviour seem relevant. Firstly, psychiatric disorder, especially depression, is associated with suicidal behaviour. ${ }^{2}$ There is evidence that there are high rates of psychiatric morbidity and neurobehavioural problems in those with head injury. ${ }^{3}$
Secondly, there are higher rates of suicide in those reporting chronic illness and unemployment. ${ }^{4}$ Feelings of hopelessness together with a poor quality of life can contribute towards suicidal behaviour.

These findings argue strongly for the importance of identifying and effectively treating depression and anxiety in people with head injury. This is no simple matter as patients are often reluctant to divulge psychological symptoms or see them as normal reactions to difficult circumstances. Linked to this is the role of rehabilitation services in providing an active, structured programme of social and occupational interventions to reduce disability.

Patients with head injury often fall into a gap between services for elderly people with stroke and younger people with psychiatric problems. All too often, patients with head injury do not receive services that can be tailored to their particular needs. An increased rate of suicide is possibly one of the most tragic and most traumatic consequences of head injury. Services for people with head injury can be improved and this should help to make their life worth living.

Department of Psychological Medicine, University of Wales, College of Medicine, Cardiff CF14 4XN, UK

wpcghl@cardiff.ac.uk

1 Teasdale W, Engberg AW. Suicide after traumatic brain injury: a population study. $\mathcal{F}$ Neurol Neurosurg Psychiatry 2001;71:436-40.

2 Barraclough B, Bunch J, Nelson B, et al. One hundred cases of suicide. Br $\mathcal{F}$ Psychiatry 1974;125:355-73.

3 Deb S, Lyons I, Koutzoukis C. Neuropsychiatric sequelae one year after a minor head injury. F Neurol Neurosurg Psychiatry 1998;65:899-902.

4 Lewis G, Sloggett A, Suicide, deprivation and unemployment. BMF 1998;317:1283-6.

\section{The entorhinal cortex in Alzheimer's disease}

In the paper by Du et al (this issue, pp 441-447) ${ }^{1}$ an evaluation of the entorhinal cortex (ERC), hippocampus (HP), and total brain tissue volumes was conducted in controls, patients with Alzheimer's disease, and non-demented subjects with a clinical dementia rating (CDR) score of 0.5 implicating mild memory impairment. Although the major finding of the study was that the HP and ERC were significantly reduced in volume for the CDR 0.5 group compared with controls, there was a large degree of overlap between the two groups and the sensitivity and specificity of correctly classifying the two groups were not improved when ERC volumes were added to HP measures alone. Postmortem studies of those with mild memory impairment and early Alzheimer's disease have implicated the ERC as the first site to carry the burden of Alzheimer's disease pathology, which then moves into the HP proper. Brain imaging studies in aging and dementia are focusing on those brain regions that might demonstrate the earliest abnormalities underlying incipient $\mathrm{AD}$. The atrophic hippocampus has yielded the best results in persons with mild impairment. Yet, with so much pathological evidence implicating the ERC as the germinal site of incipient Alzheimer's disease why has it escaped our grasp in antemortem studies?

Several factors could be responsible for the large variability of ERC imaging measures. Beyond the obvious variability of the outliner's hand and the patient's anatomy not being easily discernible due to imaging artifacts that are quite common in the medial temporal region, there is the intersubject variability of brain anatomy, normal or disease related, that confounds our outlining rules; add to this variable head positioning during scan acquisition and slice thickness that exceeds pixel size. All of these methodological issues pale if the Alzheimer's disease process itself 
is heterogeneous in its early phase with only some patients having a medial temporal disease focus. What strategies then are available to maximise the earliest disease detection?

The imaging community is now undergoing a radical advance in methodology. Studies that collapse the complexity of a brain region into a single value, such as volume, are incapable of discerning a subtle abnormality that may be present within a portion of the volume of interest. Thus, voxel based approaches are now being conducted to avoid such type II errors. Most voxel based approaches combine individual subjects within a common brain space, typically the Talairach space, for statistical analysis. Unfortunately, the aged or demented brain is not easily compatible with the Talairach space and thus anatomical mismatch occurs that hinders statistical comparisons across groups of brains. To consider this problem disease specific atlases have been created, ${ }^{2}$ with non-linear high order warping algorithms, in which surface based morphometry can be applied to detect subtle changes that escape regional volumetry. ${ }^{3}$ Extracting the unique shape changes, presumably produced by disease, encoded in the high order warping field that brings one subject into the atlas template is termed tensor mapping. Tensor mapping currently holds the greatest promise in detecting the earliest morphological disease specific change for incipient $\mathrm{AD} .^{4-6}$

Armed with a better method for image analysis is only part of the solution. The poor sensitivity/specificity of correctly classifying the mild memory group from controls when ERC volumes were added to HP measures alone, as reported in the paper by $\mathrm{Du}$ et $a l,{ }^{1}$ may result from the heterogeneity of the mildly impaired group. Not all persons with mild memory impairment have incipient Alzheimer's disease. Future studies should evaluate the ability of sensitive imaging methods to correctly identify the incipient patients with $\mathrm{AD}$ within the total group of mildly impaired persons. Now that many centres are collecting longitudinal clinical and imaging data on these "at risk" persons collaborative studies are urgently needed to increase the power of shared imaging methods across centres. Only through the sharing of common methods and data can a predictive imaging tool be validated for the identification of incipient Alzheimer's disease in the mildly impaired person who presents to a memory disorder clinic.

M S MEGA

Laboratory of Neuro Imaging, UCLA School of Medicine, Reed

Neurological Research Center, Room 4238, 710 Westwood Plaza, Los

Angeles, CA 90095-1769, USA

mega@loni.ucla.edu

1 Du A, Chuff N, Amend D, et al. Magnetic resonance imaging of the entorhinal cortex and hippocampus in mild cognitive impairment and Alzheimer's disease. F Neurol Neurosurg Psychiatry 2001;71:441-7.

2 Thompson PM, Mega MS, Toga AW. Disease-specific probabilistic brain atlases. Proceedings IEEE workshop on mathematical methods in biomedical Image Analysis. MMIBA-2000 (cat No PR00737). Los Alamitos, CA, USA: IEEE Comput Soc 2000:227-34.

3 Thompson PM, Mega MS, Woods RP, et al. Cortical change in Alzheimer's disease detected with a disease-specific population-based brain atlas. Cerebral Cortex 2001;11:1-16.

4 Mega MS, Thompson PM, Toga AW, et al. Neuroimaging in dementia. In: Mazziotta JC, Toga AW, Frackowiak R, eds. Brain mapping: the disorders. San Diego: Academic Press, 2000:217-93.

5 Csernansky JG, Wang L, Joshi S, et al. Early DAT is distinguished from aging by high-dimensional mapping of the hippocampus. Neurology 2000;55:1636-43.

6 Crum WR, Seahill RI, Fox NC. Automated hippocampal segmentation by regional fluid registration of serial MRI: validation and application in Alzheimer's disease. NeuroImage 2001;13:847-55. 